\title{
PREVALÊNCIA DE HANSENÍASE EM MULHERES PRIVADAS DE LIBERDADE NA REGIÃO METROPOLITANA DO RECIFE EM 2013
}

\section{Luiz Oscar Cardoso Ferreira}

Doutor em Medicina pela Universidade Federal de Pernambuco (UFPE); Docente Associado Universidade de Pernambuco, Faculdade de Ciências Médicas de Pernambuco, Recife (PE), Brasil.

\section{Antonio Reldismar de Andrade}

Especialização em andamento em Vigilância em Saúde Instituto Sírio-Libanês de Ensino e Pesquisa, IEP, Brasil.

E-mail: antonioreldismar@hotmail.com

Tania Maria Ferreira dos Santos

Licenciatura em Pedagogia pela Universidade Federal do Piauí (UFPI), Brasil.

\section{Mirella Cristina Bezerra de Melo}

Especialista em Saúde Coletiva pela Faculdade de Ciências Médicas da Universidade de Pernambuco (UEP), Recife (PE), Brasil.

\section{Tathiana Teles de Andrade Rocha}

Especialista em Saúde Pública pelo Programa de Residência Multiprofissional em Saúde Coletiva da Faculdade de Ciências Médicas, Universidade de Pernambuco (UEP), Recife (PE), Brasil.
RESUMO: O objetivo desse estudo é conhecer a prevalência da hanseníase e o perfil epidemiológico das mulheres privadas de liberdade na Região Metropolitana do Recife. Trata-se de um estudo de corte transversal, censitário, mediante coleta de dados por entrevistas e exames dermatológicos. Foram estudadas 1.154 mulheres, destas $81,8 \%$ são brancas, $19,5 \%$ são analfabetas, $32,4 \%$ são pertencentes às classes econômicas D e E. Detectaram-se 45 portadoras de hanseníase (forma indeterminada $=1$; tuberculóide $=16$; dimorfa $=28$ ), obtendo-se prevalência de 3,9\%. A maior prevalência ocorreu nas analfabetas $(4,9 \%)$; a unidade prisional com mais tempo de confinamento apresentou maior prevalência. A cor da pele escura é fator de risco para ocorrência da hanseníase (p-valor: $<0,001$ ); as portadoras de hanseníase eram mais jovens que as não portadoras da doença, no entanto não apresentando significância estatística. As mulheres privadas de liberdade apresentam elevada prevalência para a hanseníase, caracterizando-se por ser uma população de alta vulnerabilidade, conduzindo a necessidade de priorizar ações de vigilância à saúde nessa população.

PALAVRAS-CHAVE: Epidemiologia; Hanseníase; Mulheres; Prisões.

\section{PREVALENCE OF IMPRISONED FEMALES WITH HANSEN'S DISEASE IN THE METROPOLITAN REGION OF RECIFE IN 2013}

\begin{abstract}
The prevalence of Hansen's disease and the epidemiological profile of imprisoned females in the metropolitan region of Recife, Brazil, are investigated. Current transversal and census study was undertaken by data collection derived from interviews and dermatological tests. One thousand, one hundred and fifty-four females were studied, featuring $81.8 \%$ white people, $19.5 \%$ illiterate; $32.4 \%$ from economic classes D and E. Forty-five were suffering from Hansen's disease (undetermined $=1$; tuberculoid $=16$; dimorphous $=28$ ), with $3.9 \%$ prevalence. Greater prevalence occurred with illiterate females (4.9\%) and in those with more confinement time. Black skin is a risk factor for Hansen's disease (p-rate: <0.001) and younger females with the disease were more numerous than those without, albeit with no significantly statistical difference. Imprisoned females had a high prevalence for Hansen's disease due to high vulnerability, requiring priority in health care in this population.
\end{abstract}

KEY WORDS: Epidemiology; Hansen’s disease; females; Prisons. 


\section{INTRODUÇÃO}

A hanseníase é uma doença crônica, infectocontagiosa, que se caracteriza por manifestações dermatológicas e neurológicas. É causada por um bacilo capaz de infectar grande quantidade de pessoas (alta infectividade), entretanto poucos adoecem (baixa patogenicidade), porém possui elevado potencial incapacitante (BRASIL, 2014). O agente etiológico Mycobacterium leprae foi descrito em 1873 ea transmissão ocorre principalmente pelas vias respiratórias superiores. O período de incubação da doença é de dois a sete anos. O diagnóstico é basicamente clínico e epidemiológico. O tratamento existe há mais de 20 anos e é capaz de curar a totalidade dos casos. Os seres humanos são as únicas fontes de infecção e os doentes com as formas multibacilares (hanseníase virchowiana e dimorfa) que sem tratamento são as principais fontes de infecção da doença (BRASIL, 2010).

Predominantemente presente em países da Ásia meridional e em regiões pobres de países da América Latina, Índia e Brasil são os países com maior ocorrência da doença (WEEKLY EPIDEMIOLOGICAL RECORD, 2010).

A distribuição da hanseníase no Brasil é heterogênea e reproduz as desigualdades entre as diferentes regiões do país e indica que fatores culturais econômicos e sociais determinam sua propagação (PEREIRA et al., 2012). Dentre eles, destaca-se o encarceramento. As condições das prisões, que se configuram como ambientes insalubres, apresentam estruturas inadequadas e de grandes aglomerados de mulheres, tornando-as um local propício para a disseminação de doenças infectocontagiosas como a hanseníase, tendo como agravante ser um foco de disseminação para fora do presídio, provocada pelos profissionais que lá trabalham e pela visita de amigos e familiares (LUSTOSA et al., 2011).

Em dezembro de 2012, a população carcerária no país era de 548.003 pessoas, sendo 93,6\% (512.964) homens e 6,4\% (35.039) mulheres (BRASIL, 2012). A distribuição por sexo em Pernambuco é semelhante: em setembro de 2012 existiam 30.663 pessoas encarceradas, das quais $93,7 \%$ de homens (28.734) e 6,3\% (1.929) de mulheres (LUSTOSA et al., 2011). Entre os anos de 2002 e 2012 o aprisionamento de mulheres foi proporcionalmente três vezes maior que o sexo masculino (BRASIL, 2004). As mulheres reclusas no sistema prisional brasileiro são predominantemente jovens, mães solteiras, afrodescendentes e o envolvimento com tráfico de drogas é a principal razão da privação de liberdade (NICOLAU, 2012).

Diante da necessidade de contribuir para a estruturação da vigilância à saúde nos presídios considerando o contexto social, econômico e cultural da população carcerária, pretendeu-se conhecer a prevalência da hanseníase em mulheres privadas de liberdade na Região Metropolitana do Recife.

\section{METODOLOGIA}

Trata-se de um estudo clínico e epidemiológico, transversal, realizado em 2013, com mulheres privadas de liberdade em duas unidades prisionais. A Colônia Penal Feminina do Recife tem capacidade para 150 reeducandas, abriga cerca de 700 mulheres, distribuídas em 33 celas. A Colônia Penal Feminina de Abreu e Lima albergava cerca de 500 mulheres em 40 celas, programadas para abrigar apenas cinco mulheres em cada uma.

A população do estudo foi o universo de mulheres presente nos dois presídios no momento da coleta de dados, que foi realizada por entrevista face a face para identificação de perfil socioepidemiológico e exame clínico. Incialmente foram usadas para a triagem das mulheres com manchas as fichas de autoimagem, no entanto, após uma baixa adesão ao método, distribuiu-se para todas as mulheres uma ficha de autopreenchimento para identificação de manchas cutâneas, entretanto nem todas as fichas foram devolvidas. Posteriormente, na ocasião em que a profissional de saúde estava realizando os exames dermatológicos, estendeu-se o método para todas as mulheres que independentemente de terem entregues as fichas de autoimagem ou não deveriam ser submetidas ao exame clínico-dermatológico de sensibilidade cutânea nos locais das manchas. $\mathrm{O}$ "Critério Brasil - ABEP" da Associação Brasileira de Empresas de Pesquisa (2012) foi utilizado para a classificação 
econômica e a cor da pele foi autorreferida. A taxa de prevalência foi calculada pela razão entre o número de casos de hanseníase e a população do presídio. $\mathrm{O}$ processamento e análise dos dados foram realizados com o uso do software Epi - Info, verão 6.04 (CENTERS FOR DISEASE CONTROL AND PREVENTION/OMS).

O presente estudo é parte da Pesquisa "Perfil Clínico e Epidemiológico da Tuberculose, Hanseníase e Infecções Sexualmente Transmissíveis em presidiárias da Região Metropolitana do Recife - 2013”, patrocinada pela SVS/MS, tendo sido aprovada por Comitê de Ética (Parecer $\mathrm{n}^{\circ}$ 425013). As entrevistadas participaram livremente do estudo, tendo sido fornecidas todas as informações necessárias previamente e após assinatura do Termo de Consentimento Livre e Esclarecido (TCLE).

\section{RESULTADOS}

Estudaram-se 1.154 mulheres, sendo 57,7\% (n $=667) \mathrm{da}$ Colônia Penal Feminina do Recife (CPFR) e $42,3 \%(\mathrm{n}=487)$ da Colônia Penal Feminina de Abreu e Lima (CPFAL).

Relataram serem portadores de manchas na pele 369 mulheres, produzindo uma frequência de $32,0 \%(369 / 1.154)$ de manchas. Dessas diagnosticaramse 45 portadoras de Hanseníase (forma indeterminada: $\mathrm{HI}=1$; forma tuberculóide: $\mathrm{HT}=16$; forma dimorfa: $\mathrm{HD}=28$ ), obtendo-se uma prevalência de $12,2 \%$ entre as portadoras de mancha (45/369). Admitindo-se que as mulheres que não referiram apresentar manchas não eram portadoras da doença, pode-se inferir que a prevalência de hanseníase na população estudada foi de 3,9\% (45/1.154). Salienta-se que apenas sete mulheres tinham diagnóstico prévio de hanseníase.

A mulher mais jovem tinha 18,3 anos e a mais idosa 83,6 anos (média de 31,9, DP \pm 10,0). A faixa etária mais frequente foi de 20 a 30 anos e 19,2\% das mulheres referiram ter cor branca. A situação conjugal mais comum foi a casada/mora junto. Chama atenção que 31,2\% referiram não ter religião, percentual mais elevado que as outras religiões isoladamente, sendo inferior apenas à católica (Tabela 1).
Tabela 1. Características demográficas de mulheres privadas de liberdade na Região Metropolitana do Recife. Pernambuco, Brasil, 2013

\begin{tabular}{|c|c|c|}
\hline Variáveis Sociodemográficas & $\mathbf{n}$ & $\%$ \\
\hline \multicolumn{3}{|l|}{ Faixa Etária $(n=1.147)$} \\
\hline $18-20$ & 50 & 4,4 \\
\hline $20-30$ & 551 & 48,0 \\
\hline $30-40$ & 307 & 26,8 \\
\hline $40-50$ & 163 & 14,2 \\
\hline $50-60$ & 65 & 5,7 \\
\hline$>60$ & 11 & 1,0 \\
\hline \multicolumn{3}{|l|}{ Cor $(n=1.154)$} \\
\hline Negra & 118 & 10,2 \\
\hline Parda & 422 & 36,6 \\
\hline Amarela & 28 & 2,4 \\
\hline Branca & 222 & 19,2 \\
\hline Indígena & 4 & 0,3 \\
\hline Outros & 360 & 31,2 \\
\hline \multicolumn{3}{|l|}{ Estado Civil $(\mathrm{n}=1.154)$} \\
\hline Solteira & 463 & 40,1 \\
\hline Casada/mora junto & 548 & 47,5 \\
\hline União Estável & 55 & 4,8 \\
\hline Viúva & 76 & 6,6 \\
\hline Outros & 12 & 1,0 \\
\hline \multicolumn{3}{|l|}{ Religião $(n=1.154)$} \\
\hline Católica & 491 & 42,6 \\
\hline Evangélica & 264 & 22,9 \\
\hline Kardecista & 12 & 1 \\
\hline Umbanda & 12 & 1 \\
\hline Outros & 16 & 1,3 \\
\hline Sem Religião & 359 & 31,2 \\
\hline
\end{tabular}

Quanto ao tempo de privação de liberdade registrou-se que, em média, elas estavam há 21,9 meses $(\mathrm{DP} \pm 22,3)$ encarceradas. Na CPFAL as mulheres estão há mais tempo quando comparado à CPFR $(36,7 \pm 24,6$ meses $\times 11,0 \pm 12,0$ meses).

Cerca de $20 \%$ das reeducandas são analfabetas. A escolaridade mais frequente foi ensino primário completo (corresponde até $4^{\mathrm{a}}$ série ou $5^{\circ}$ ano)/ginásio incompleto. Quanto à classe econômica foi possível observar que $32,4 \%$ pertencem à classe $\mathrm{D}$ e $\mathrm{E}$. A classe $\mathrm{C}$ foi a mais prevalente com 56,5\% (Tabela 2). 
Tabela 2. Características socioeconômicas de mulheres privadas de liberdade na Região Metropolitana do Recife. Pernambuco, Brasil, 2013

\begin{tabular}{lcc}
\hline Variáveis Sociodemográficas & $\mathbf{n}$ & \% \\
\hline Ler/Escrever um Bilhete $(\mathbf{n}=\mathbf{1 . 1 5 4})$ & & \\
\hline Sabe & 929 & 80,5 \\
Não Sabe & 225 & 19,5 \\
\hline Escolaridade(n=1.153) & & \\
\hline Alfabeto/Primário incompleto & 326 & 28,3 \\
Primário completo/Ginásio incompleto & 446 & 38,7 \\
Ginásio completo/Colegial incompleto & 236 & 20,5 \\
Colegial completo/Superior incompleto & 133 & 11,5 \\
Superior completo & 12 & 1,0 \\
\hline Critério Econômico (n=1.154) & & \\
\hline A1 & 3 & 0,3 \\
A2 & 10 & 0,9 \\
B1 & 31 & 2,7 \\
B2 & 84 & 7,3 \\
C1 & 212 & 18,4 \\
C2 & 440 & 38,1 \\
D & 305 & 26,4 \\
E & 69 & 6,0 \\
\hline
\end{tabular}

No que se refere à percepção das mulheres privadas de liberdade sobre sua saúde (Tabela 3), verificase que foi maior a frequência do relato de "saúde boa". Apenas 12,6\% referiram "saúde ruim" ou "saúde muito ruim".

Tabela 3. Distribuição da percepção de saúde de mulheres privadas de liberdade na Região Metropolitana do Recife, Pernambuco, Brasil, 2013

\begin{tabular}{lcc}
\hline Variáveis & n & $\%$ \\
\hline Saúde & & \\
Muito Boa & 106 & 9,2 \\
Boa & 526 & 45,6 \\
Regular & 364 & 31,5 \\
Ruim & 100 & 8,7 \\
Muito Ruim & 45 & 3,9 \\
\hline
\end{tabular}

Não se obteve diferença estatisticamente significante quando se observou a idade em relação à doença (Tabela 4). Nas mulheres não portadoras de hanseníase a idade foi mais elevada em relação às portadoras $(32,0 \pm 10,0 \times 30,0 \pm 8,9)$. Também não se observou diferença estatisticamente significante entre saber ou não saber ler e a prevalência da doença, embora que nas analfabetas a prevalência foi maior que nas alfabetizadas $(4,9 \% \times 3,6 \%)$.

Tabela 4. Associações entre variáveis e o diagnóstico de hanseníase em mulheres privadas de liberdade na Região Metropolitana do Recife, Pernambuco, Brasil, 2013

\begin{tabular}{|c|c|c|c|c|c|c|c|}
\hline \multirow[t]{3}{*}{ Variáveis } & \multicolumn{4}{|c|}{ Diagnóstico de Hanseníase } & \multirow{3}{*}{ OR } & \multirow{3}{*}{ IC $95 \%$} & \multirow{3}{*}{$p$-valor } \\
\hline & \multicolumn{2}{|c|}{ SIM } & \multicolumn{2}{|c|}{ NÃO } & & & \\
\hline & $\mathbf{N}$ & $\%$ & $\mathrm{~N}$ & $\%$ & & & \\
\hline Faixa Etária & & & & & & & 0,43 \\
\hline $18 \cdot 20$ & 4 & 8,0 & 46 & 92,0 & & & \\
\hline $20-30$ & 24 & 4,4 & 527 & 95,6 & & & \\
\hline $30-40$ & 9 & 2,9 & 298 & 97,1 & & & \\
\hline $40-50$ & 6 & 3,7 & 157 & 96,3 & & & \\
\hline $50-60$ & 1 & 1,5 & 64 & 98,5 & & & \\
\hline 60 a mais & 0 & 0,0 & 11 & 100,0 & & & \\
\hline \multirow[t]{2}{*}{ Ler/Escrever um Bilhete } & & & & & 0,72 & $0,34-$ & 0,45 \\
\hline & & & & & & 1,54 & \\
\hline Não sabe & 11 & 4,9 & 214 & 95,1 & & & \\
\hline Sabe & 33 & 3,6 & 896 & 96,4 & & & \\
\hline Escolaridade & & & & & & & 0,46 \\
\hline Analfabeto $/<3^{\mathrm{a}}$ série & 16 & 4,9 & 310 & 95,1 & & & \\
\hline Até $4^{\mathrm{a}}$ série Fundamental & 17 & 3,8 & 429 & 96,2 & & & \\
\hline Fundamental completo & 5 & 2,1 & 231 & 97,9 & & & \\
\hline Médio completo & 6 & 4,5 & 127 & 95,5 & & & \\
\hline Superior completo & 0 & 0,0 & 12 & 100,0 & & & \\
\hline Cor & & & & & & & $<0,001$ \\
\hline Negra & 6 & 5,1 & 112 & 94,9 & & & \\
\hline Parda & 17 & 4,1 & 405 & 96,2 & & & \\
\hline Branca & 3 & 1,4 & 219 & 98,6 & & & \\
\hline Amarela & 2 & 7,1 & 26 & 92,9 & & & \\
\hline Indígena & 0 & 0,0 & 4 & 100,0 & & & \\
\hline Outros & 16 & 4,4 & 344 & 95,6 & & & \\
\hline Critérios Econômicos & & & & & & & 0,31 \\
\hline $\mathrm{A} 1+\mathrm{A} 2+\mathrm{B} 1+\mathrm{B} 2$ & 2 & 1,6 & 126 & 98,4 & & & \\
\hline $\mathrm{C} 1+\mathrm{C} 2$ & 25 & 3,8 & 627 & 96,2 & & & \\
\hline $\mathrm{D}+\mathrm{E}$ & 17 & 4,5 & 357 & 95,5 & & & \\
\hline
\end{tabular}

Na Tabela 4 está demonstrado que as mulheres portadoras de hanseníase são menos favorecidas socialmente, pois apresentam maior proporção de analfabetas e pertencendo às classes sociais mais baixas, mesmo considerando que essas diferenças não foram estatisticamente significantes. Já com a condição de raça/ cor se observou diferenças estatisticamente significantes, o que conduz a afirmação que ser de cor não branca é fator de risco para a doença.

Não se observou diferença entre pertencer à unidade prisional e ser portadora de hanseníase embora 
que no presídio com mais tempo de privação de liberdade, o CPFAL, cuja média foi de 36,7 meses ( $\mathrm{DP}=24,6$ meses) detectou-se uma prevalência maior, $4,1 \%$ que no presídio de menor duração média de privação de liberdade, o CPFR (11,0 $0 \pm 12,0$ meses), cuja prevalência foi de 3,6\% (Tabela 5). Considerando-se o tempo de privação de liberdade entre as mulheres portadoras de hanseníase registrou-se uma média de 21,1 meses $(\mathrm{DP}=19,3)$ comparadas com 22,0 $(\mathrm{DP}=22,4)$ meses entre as não portadoras da enfermidade, entretanto essa diferença não foi estatisticamente significante $(t=0548 ; \mathrm{p}=0,197)$. Focando apenas 45 mulheres portadoras de hanseníase observou-se uma média de 18,2 ( $\mathrm{DP}=15,2)$ meses de privação de liberdade; quatorze mulheres, isto é, 32,6\% (14/43) estavam no presídio há mais de 24 meses.

Tabela 5. Associações entre variáveis e o diagnóstico de hanseníase em mulheres privadas de liberdade na Região Metropolitana do Recife, Pernambuco, Brasil, 2013

\begin{tabular}{|c|c|c|c|c|c|c|c|}
\hline \multirow[t]{3}{*}{ Variáveis } & \multicolumn{4}{|c|}{ Diagnóstico de Hanseníase } & \multirow{3}{*}{ OR } & \multirow{3}{*}{ IC $95 \%$} & \multirow{3}{*}{$p$-valor } \\
\hline & \multicolumn{2}{|c|}{ SIM } & \multicolumn{2}{|c|}{ NÃO } & & & \\
\hline & $\mathbf{N}$ & $\%$ & $\mathbf{N}$ & $\%$ & & & \\
\hline Colônia Penal & & & & & 0,87 & $0,46-1,67$ & 0,65 \\
\hline CPFAL & 20 & 4,1 & 467 & 95,9 & & & \\
\hline CPFR & 24 & 3,6 & 643 & 96,4 & & & \\
\hline Percepção da Saúde & & & & & $\cdot$ & - & 0,03 \\
\hline Muito Boa & 2 & 1,9 & 104 & 98,1 & & & \\
\hline Boa & 16 & 3,0 & 510 & 97,0 & & & \\
\hline Regular & 14 & 3,8 & 350 & 96,2 & & & \\
\hline Ruim & 9 & 9,0 & 91 & 91,0 & & & \\
\hline Muito Ruim & 3 & 6,7 & 42 & 93,3 & & & \\
\hline
\end{tabular}

Sobre a percepção do estado de saúde se observou que as mulheres portadoras de hanseníase consideraram sua "saúde ruim" e "saúde muito ruim" proporcionalmente mais elevada que as não portadoras da doença, mas as diferenças não se mostraram estatisticamente significantes.

\section{DISCUSSÃO}

As mulheres privadas de liberdade da Região Metropolitana do Recife não diferem das mulheres encarceradas em outros presídios brasileiros: são jovens, afrodescendentes, pouco escolarizadas e de baixa condição socioeconômica e encontram-se albergadas nas mesmas condições de superlotação (NICOLAU, 2012).

Foram encontradas 45 mulheres portadoras de hanseníase em uma população de 1.154 mulheres. Esta prevalência de 3,9\% é demasiadamente elevada quando comparada com a taxa de detecção registrada em Pernambuco pelo Sistema Nacional de Agravos de Notificação (SINAN) de 29,2 por 100 mil habitantes. Se a taxa do SINAN em Pernambuco fosse aplicada nessa população se estimaria encontrar apenas 0,33 casos nas 1.154 mulheres, quando foram encontradas 45 portadoras da doença, isto é, cerca de mais de 100 vezes, mesmo considerando-se que foi realizada uma busca ativa nas unidades prisionais (SECRETARIA ESTADUAL DE SAÚDE DE PERNAMBUCO, 2013). Não se encontraram dados na literatura sobre ocorrência de hanseníase em população privada de liberdade.

Entre escolares de 5 a 19 anos de idade na cidade de Paracatu, em Minas Gerais, nos anos de 2004 a 2006 quando foram examinados 16.623 mulheres de áreas urbana e rural, os pesquisadores encontraram 68 casos de hanseníase, isto é, 0,41\% (BRASIL, 2011). Isto significa que na população presidiária aqui estudada o valor foi cerca de 10 vezes maior, entretanto deve-se levar em consideração, além da idade dos escolares, que os métodos de diagnóstico podem não ser comparáveis. Mesmo assim, se observa valores ainda bastante elevados na população encarcerada.

Como o período de incubação da doença é longo, com intervalo de dois a cinco anos (MINISTÉRIO DA SAÚDE, 2014), e considerando-se que apenas duas mulheres estavam no presídio há mais de 48 meses, pode-se supor que a infecção ocorreu antes mesmo do confinamento. Como a hanseníase tem uma marca social forte e essas mulheres pertencem às classes socioeconômicas mais desfavorecidas o risco epidemiológico se traduz majoritariamente por transmitir o bacilo entre elas para anos depois a doença se apresentar nas formas clínicas.

Mais da metade das mulheres apresentou a forma de hanseníase dimorfa (28/45) o que quer dizer que o bacilo estava presente e, levando-se em conta a elevada infectividade e o fato da aglomeração e contato diuturno, pode-se supor que o ambiente prisional representa um elevado risco para a infecção pelo bacilo de Hansen.

Considerando-se que apenas 07 das 45 mulheres 
portadoras do mal de Hansen tinham diagnóstico da doença, denota-se que o Plano Nacional de Saúde no Sistema Penitenciário, instituído pela portaria interministerial $\mathrm{n}^{\circ} 1777 / 2003$, não está sendo observado, pois ele preconiza a atenção à dermatologia sanitária, com ênfase na hanseníase, mediante a busca ativa de casos e o diagnóstico por meio clínico-epidemiológico, além de exame de comunicantes dos familiares do caso (BRASIL, 2003). O predomínio de mulheres analfabetas e de baixa escolaridade entre os portadores de hanseníase demonstra uma importante variável a ser levada em consideração pelos serviços de saúde no planejamento e desenvolvimento das atividades, principalmente na elaboração de ações educativas que visem a promoção da saúde e formas de prevenção e tratamento da doença, inclusive as incapacidades (FERREIRA, 2008).

\section{CONCLUSÕES}

O estudo evidencia a necessidade do fortalecimento da vigilância à saúde dentro dos presídios femininos. A realização de exame clínico-epidemiológico nas mulheres no momento em que são introduzidas no sistema prisional, além da importância de se estabelecer na rotina dos profissionais de saúde exames periódicos visando à detecção precoce de novos casos de hanseníase, são pontos cruciais nesse processo. Para isto se faz necessário promover capacitações e cursos de atualização para os profissionais de saúde dos presídios levando-se em conta a vulnerabilidade dessa população para as doenças infectocontagiosas.

Conjuntamente e com mesma magnitude, a adoção de ações de educação em saúde para as reeducandas, que se fundamentem em estratégias que valorizem a vida social, cultural, econômica e as crenças com objetivo de resgatar a dignidade humana e permitir a construção da autonomia e do autocuidado, se fazem importantes na promoção de vidas mais saudáveis no cárcere e consequentemente fora dele.

O presente estudo contribui para realização de outras pesquisas relacionadas ao tema, além de subsidiar a tomada de decisão dos gestores a partir do conhecimento desse perfil epidemiológico.

\section{REFERÊNCIAS}

AMARAL, E. P.; LANA, F. C. F. Análise espacial da Hanseníase na microrregião de Almenara, MG, Brasil. Rev. Bras. Enferm, Brasília, v. 61, n. esp, p. 701-707, 2008. Disponível em: < http://www.scielo.br/pdf/reben/ v61nspe/a08v61esp.pdf> . Acesso em: 12 ago. 2014.

ASSOCIAÇÃO BRASILEIRA DE EMPRESAS DE PESQUISA - ABEP. Critério de Classificação Econômica Brasil: Base LSE. São Paulo: ABEP, 2012.

BRASIL. Ministério da Justiça. Departamento Penitenciário Nacional. Relatório Estatística da População Carcerária. [s.l.; s.n.], 2012.

BRASIL. Ministério da Justiça. Departamento Penitenciário Nacional. Diretoria de Políticas Penitenciárias. Coordenação da Comissão Especial Projeto Mulheres. Mulheres Presas: Dados Gerais/Projeto Mulheres DEPEM. Brasília: Ministério da Justiça, 2011.

BRASIL. Presidência da República. Lei $\mathrm{n}^{0} 7.210$, de 11 de julho de 1984. Institui a Lei de Execução Penal. Diário Oficial da República Federativa do Brasil, Brasília, 13 jul. 1984, Seção 1:10227.

BRASIL. Ministério da Saúde e Ministério da Justiça. Portaria Interministerial $\mathrm{n}^{0} 1777$, de 09 de dezembro de 2003. Aprova o Plano Nacional de Saúde no Sistema Penitenciário.

BRASIL. Ministério da Saúde. Portaria no 3.125, de 07 de outubro de 2010. Aprova as Diretrizes para Vigilância, Atenção e Controle da hanseníase sob gestão da Secretaria de Vigilância em Saúde. Diário Oficial da República Federativa do Brasil, Brasília, 15 out. 2010. Seção 1:55.

BRASIL. Ministério da Saúde. Secretaria de Atenção à Saúde. Departamento de Ações Programáticas Estratégicas. Plano Nacional de Saúde no Sistema Penitenciário. 3. ed. Brasília: Ministério da Saúde, 2004.

BRASIL. Ministério da Saúde. Secretaria de Vigilância em Saúde. Guia de Vigilância em Saúde. Brasília: Ministério da Saúde, 2014. 
PREVENTION/OMS - Organização Mundial da Saúde. Software Epi Info 6.04.

FERREIRA, I. N. Busca ativa de hanseníase na população escolar e distribuição espacial da endemia no município de Paracatu - MG (2004 a 2006). 2008. 127f. Tese (Doutorado em Ciências da Sa Brasília: Universidade de Brasília, Brasília, DF. 2008.

LUSTOSA, A. A.; NOGUEIRA, L. T.; PEDROSA, J. I. S.; TELES, J. B. M; CAMPELO, V. The impact of leprosy on health-related quality of life. Rev Soc Bras Med Trop., Uberaba, v. 44, n. 5, sept./oct. 2011. Disponível em: $<$ http://www.scielo.br/scielo.php? > . Acesso em: 18 ago. 2014

NICOLAU, A. I. O.; RIBEIRO, S. G.; LESSA, P. R. A.; MONTE, A. S.; FERREIRA, R. C. N.; PINHEIRO, A. K. B. Retrato da realidade socioeconômica e sexual de mulheres presidiárias. Acta paul enferm., São Paulo, v. 25, n. 3, 2012. Disponível em: $<$ http://www.scielo.br/scielo.php?script $=$ sci arttext\&pid $=$ S0103-21002012000300011 $>$. Acesso em: 18 ago. 2014.

PEREIRA, D. L.; BRITO, L. M.; NASCIMENTO, A. H.; RIBEIRO, E. L.; LEMOS, K. R. M.; ALVES, J. N.; BRANDÃO, L. C. G. Estudo da Prevalência das Formas Clínicas da Hanseníase na Cidade de Anápolis-GO. Ensaios e Ciência: Ciências Biológicas, Agrárias e da Saúde, v. 16, n. 1, p. 55-67, 2012. Disponível em: <http://www. redalyc.org/pdf/260/26025372004.pdf $>$. Acesso em: 10 mai. 2014.

SECRETARIA DE DESENVOLVIMENTO SOCIAL E DIREITOS HUMANOS (PE). Secretaria Executiva de Ressocialização - SERES. Relatório do Fluxo Migratório das Unidades Prisionais do Estado de Pernambuco. Recife: Secretaria de Desenvolvimento Social e Direitos Humanos, 2012.

SECRETARIA DE ESTADO DA SAÚDE DO ESTADO DE SÃO PAULO. O HIV e a Sífilis no Sistema Prisional Feminino do Estado de São Paulo. São Paulo: [s.n.], 2013.
SECRETARIA ESTADUAL DE SAÚDE DE PERNAMBUCO. Secretaria Executiva de Vigilância em Saúde. Recife: [s.n.], 2013.

WEEKLY EPIDEMIOLOGICAL RECORD. Relevé épidémiologique hebdomadaire. Global leprosy situation, v. 35, n. 85, p. 337-348, 2010. Disponível em: $<$ http://www.who.int/wer/2010/wer8535.pdf>. Acesso em: 11 ago. 2014.

Recebido em: 20 de março de 2016 Versão final recebida em: 03 de maio de 2016 Aceito em: 22 de maio de 2016 